\title{
Estimación de parámetros
}

Autor

Romero Saldaña M

Enfermero Especialista en Enfermería del Trabajo

"Quien no sabe lo que busca, no ve lo que encuentra" Claude Bernard

\section{Estadística Inferencial o estadística Analítica}

Imaginemos que deseamos conocer la media de edad de la población de nuestra empresa que tiene un total de 877 trabajadores/as. Para conseguir nuestro propósito, podríamos llevar a cabo dos acciones: por un parte, podríamos encuestar a toda la población trabajado- ra y a partir de estos datos hallar su media; o bien, podríamos tomar una muestra (de forma aleatoria y estratificada por edad y sexo), por ejemplo de 100 trabajadores/as, a continuación obtener su media y a partir de la misma, estimar o inferir la media de edad de la población total.

La inferencia permite obtener el valor de los parámetros (población) a partir del resultado observado en los estadísticos (muestra), todo ello, dentro de un determinado rango de valores, al cual denominaremos intervalo de confianza, y con una alta probabilidad de acierto, que se asigna como el nivel de seguridad o significación estadística.

Figura 1. Estimación de parámetros.

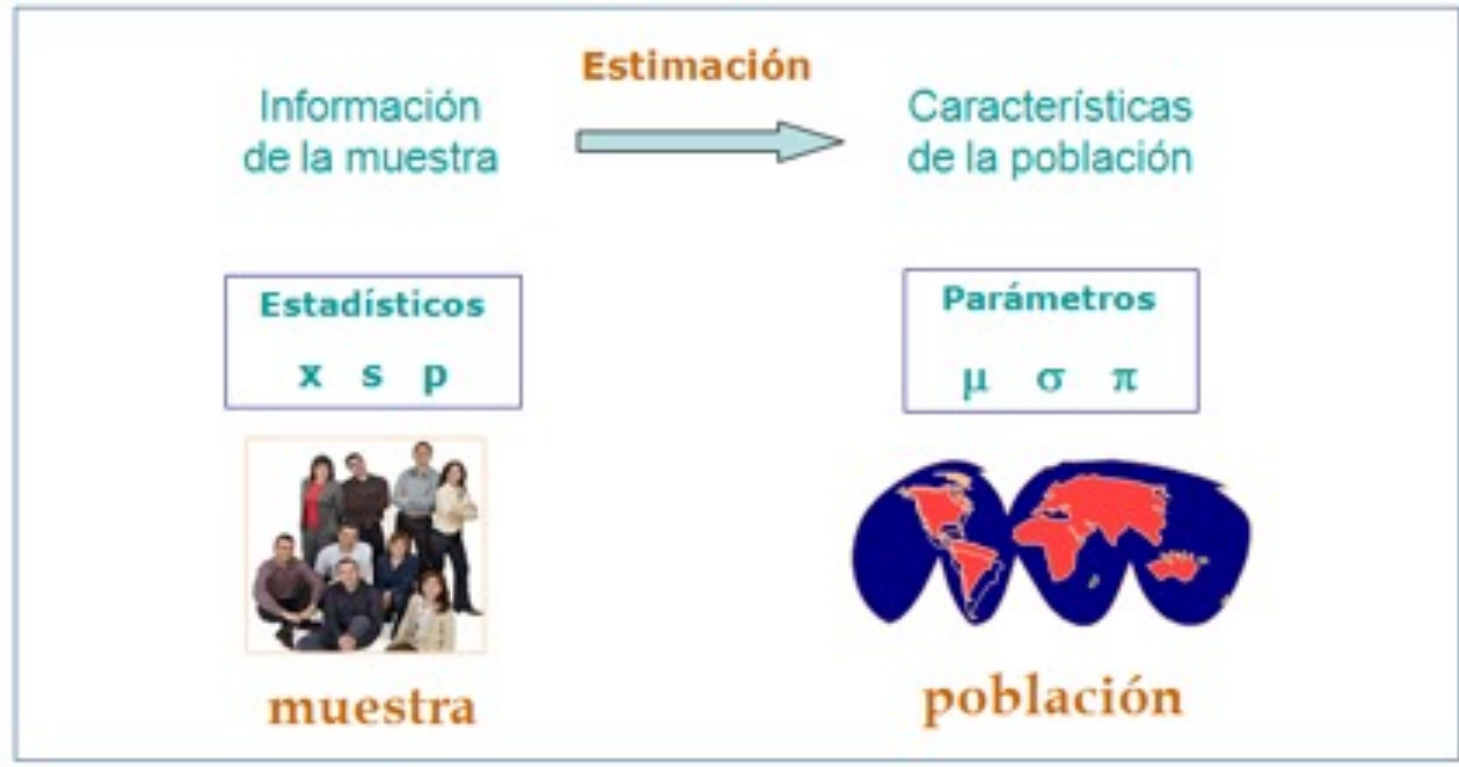


Comencemos por definir algunos conceptos de interés:

- Estadística Inferencial o Analítica: Su objetivo es "inferir", o sea, establecer conclusiones sobre una población a partir de los resultados obtenidos de una muestra, todo ello, con un determinado nivel de seguridad o intervalo de confianza.

- Población: Conjunto de personas, sujetos o unidades que presentan una característica común.

- Muestra: Subconjunto extraído y seleccionado de una población a la que representa. Podemos distinguir:

- Muestra independiente: o datos independientes, son aquellos obtenidos tras una única observación.

- Muestra apareada: o datos apareados, aquellos que comparan el mismo grupo de sujetos en dos momentos del tiempo (por ejemplo antes y después de una intervención), o bien son grupos muy relacionados entre sí.

- Estadístico: Índice que representa una información de la muestra estudiada. Suelen expresarse mediante letras del alfabeto latino. Ej. Desviación estándar de la muestra (s), media aritmética (x).

- Parámetro: Cada uno de los estadísticos que tras inferirse, nos proporcionan información sobre la población. A diferencia de los estadísticos, éstos se representan mediante letras del alfabeto griego. Ej. Desviación estándar de la población $(\sigma)$, media aritmética $(\mu)$.

- Intervalo de Confianza: Es un rango de valores (calculado en una muestra) en el cual se encuentra el verdadero valor del parámetro, con una probabilidad determinada. La probabilidad de que el verdadero valor del parámetro se encuentre en el intervalo construido se denomina nivel de confianza (1- $\alpha$ ). La probabilidad de equivocarnos se llama nivel de significancia $(\boldsymbol{\alpha})$. Generalmente se construyen intervalos de confianza con $1-\alpha=95 \%$ (o significancia $\alpha=5 \%$ ). En un intervalo de confianza encontraremos por tanto dos valores o límites de confianza: el valor más pequeño, denominado límite inferior, y el valor mayor, o límite superior.

\section{Estimación de parámetros}

Para estimar un parámetro, lo primero que debemos conocer es su error estándar (EE) o también conocido como error típico.

Se define el EE como la desviación estándar de una distribución de dicho estadístico. Ej. El EE de la media, será la desviación estándar de una distribución formada $\mathrm{n}$ medias aritméticas obtenidas del análisis de $\mathrm{n}$ muestras de una población. De igual forma, el EE de un porcentaje, es la desviación estándar de una distribución formada $\mathrm{n}$ porcentajes resultantes de la observación de $\mathrm{n}$ muestras de esa población.

En definitiva, el EE nos proporciona información sobre la dispersión que presentaría, tras una hipotética repetición del estudio, el estadístico observado.

Matemáticamente, el EE se obtiene dividiendo la desviación estándar del estadístico entre la raíz cuadrada del tamaño muestral. Su fórmula es la siguiente:

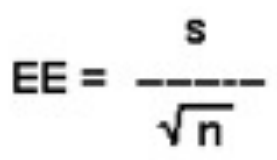

Cuanto más pequeño sea el EE más pequeña será la variación de los estadísticos (media, porcentaje, etc.); es decir, más preciso resulta el estudio. De esta ecuación se deduce que al aumentar el tamaño muestral (n), disminuye el EE, y viceversa.

La figura 2 ilustra los pasos a seguir para la obtención del EE:

1. Tenemos una población o universo.

2. De ella se selecciona $n$ muestras $\left(m_{1}, m_{2}, m_{3}\right.$, $\left.\mathrm{m}_{4} \ldots . . \mathrm{m}_{\mathrm{n}}\right)$.

3. De cada una de estas muestras se obtiene su media correspondiente, y a partir de las mimas, se elabora la distribución de medias.

4. Finalmente, el EE de la media, será la desviación típica de esta distribución de medias.

Ni que decir tiene que para calcular el EE de un parámetro no tenemos por que hacer todos estos pasos, bastaría sencillamente con aplicar la fórmula descrita anteriormente. 
Figura 2. El error estándar de la media.
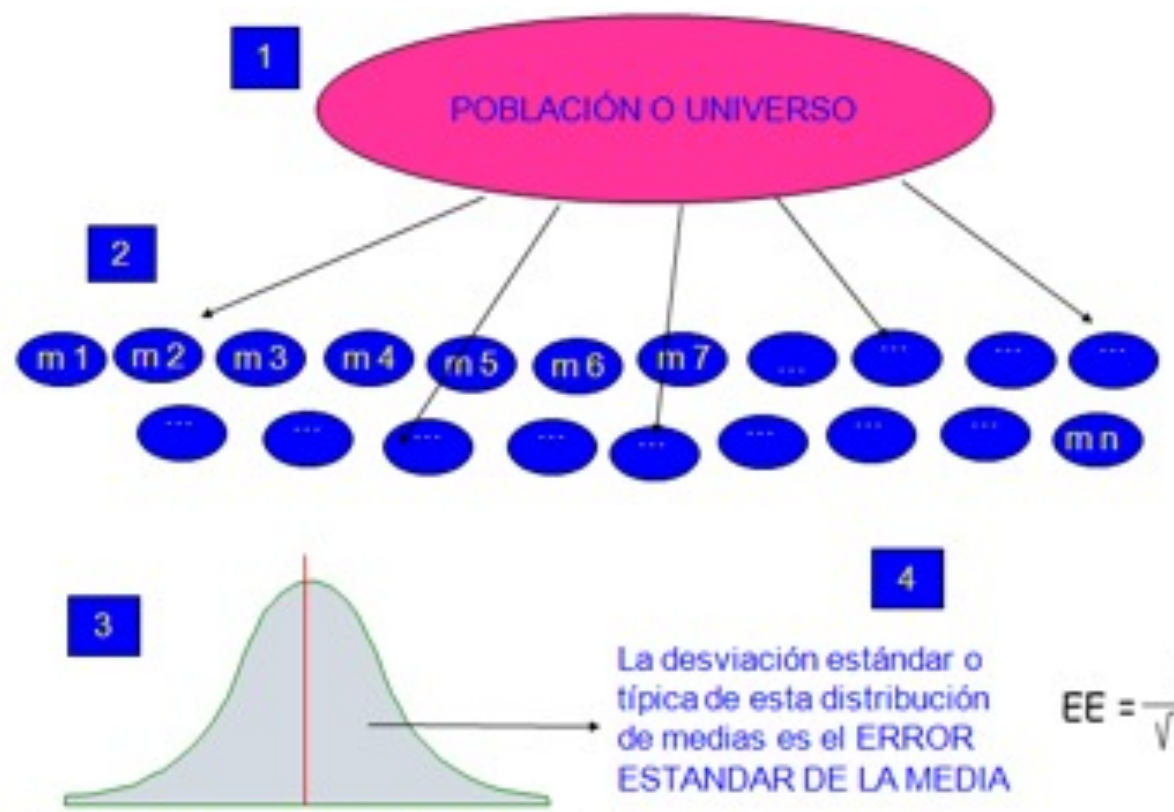

4

Distribución de las medias aritméticas de las muestras

\subsection{Estimación de la media}

Para estimar la media de una población $(\mu)$ a partir de la media obtenida de una muestra $(\mathrm{X})$, será necesario en primer lugar, conocer su EE.

El EE de la media, EEM, se define como la desviación estándar de sucesivas medias obtenidas de varias muestras de esta población, y se calcula mediante la siguiente fórmula:

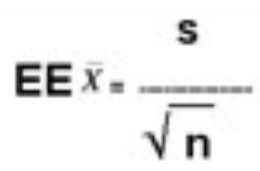

donde "s" es la desviación típica de la muestra analizada y "n" su tamaño muestral.

Una vez determinado el EE de la media (EEM), el segundo paso será concretar el intervalo de confianza (IC). Recordemos que el IC supone el rango de valores dentro del cual existe una probabilidad concreta de encontrarse el parámetro estimado.

Esta probabilidad o seguridad puede ser del 95\%, 99\% ó 99,9\%, aceptando implícitamente un error de equivocarnos del 5\%,1\% y $0,1 \%$ respectivamente. Por último, se considera apropiado el nivel de confianza del $95 \%$, necesitando para ello, sumar y restar 1,96 veces el EEM a la media aritmética.

$$
\text { IC }(\alpha=5 \%) \text { ó } p<0,05=X \pm 1,96 \text { EEM }
$$

Un IC con una seguridad del $95 \%$, que es lo mismo decir con un error menor del 5\% $(\mathrm{p}<0,05)$, significa que si el estudio se repitiera 100 veces, en el $95 \%$ de los mismos, la media aritmética de la población estaría comprendida dentro de este intervalo de confianza.

$\mathrm{Si}$ queremos determinar el IC con una seguridad del $99 \%$, o lo que es lo mismo, un error inferior al $1 \%$, habrá que sumar y restar a la media 2,57 veces el EEM.

\section{Estimación de la media para muestras pequeñas}

Todo lo visto anteriormente, se aplica cuando el tamaño muestral es mayor de 30 observaciones. Entonces, se considera que los datos de la muestra siguen una distribución normal. Sin embargo, a veces podemos encontrar problemas para conseguir muestras con más de 30 sujetos, observaciones, datos, etc. Estas muestras se consideran pequeñas y sus datos siguen una distribución denominada "t de Student" para muestras pequeñas. 
Figura 3. Representación gráfica del concepto de estimación.

Ser un estimador adecuado no significa ....

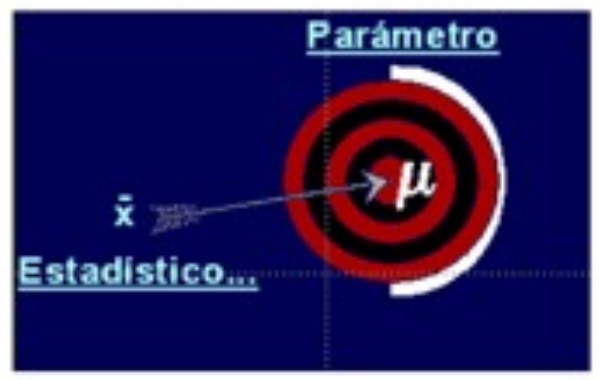

significa ...

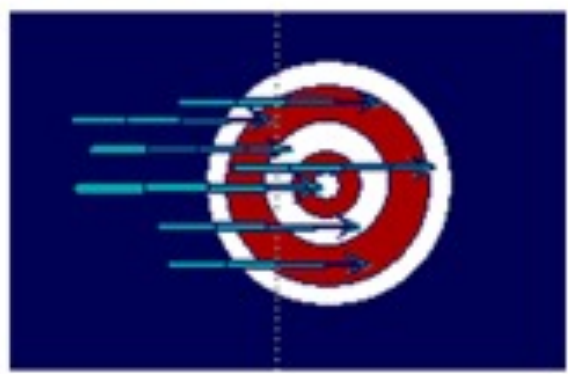

... manejo de la incertidumbre y de la imprecisión

Figura 4. Tabla de la distribución t de Student.

\begin{tabular}{|c|c|c|c|c|c|c|}
\hline $\begin{array}{l}\text { Grados de } \\
\text { libertad }\end{array}$ & 0.25 & 0.1 & 0.05 & 0.025 & 0.01 & 0.005 \\
\hline 1 & 1.0000 & 3.0777 & 6.3137 & 12.7062 & 31.8210 & 63.6559 \\
\hline 2 & 0.8165 & 1.8850 & 2.9200 & 4.3027 & 6.8645 & 8.9250 \\
\hline 3 & 0.7849 & 1.0377 & 2.3634 & 3.1824 & 4.5407 & 5.8408 \\
\hline 4 & 0.7407 & 1.5332 & 2.1318 & 2.7765 & 3.7469 & 4.6041 \\
\hline 5 & 0.7267 & 1.4759 & 2.0150 & 2.5706 & 3.3649 & 4.0321 \\
\hline 6 & 0.7176 & 1.4398 & 1.9432 & 2.4469 & 3.1427 & 3.7074 \\
\hline 7 & 0.7111 & 1.4149 & 1.8948 & 2.3646 & 2.9979 & 3.4995 \\
\hline 8 & 0.7064 & 1.3968 & 1.8695 & 2.3060 & 2.8965 & 3.3554 \\
\hline 9 & 0.7027 & 1.3830 & 1.8331 & 2.2622 & 2.8214 & 3.2498 \\
\hline 10 & 0.6998 & 1.3722 & 1.8125 & 2.2281 & 2.7638 & 3.1693 \\
\hline 11 & 0.6974 & 1.3634 & 1.7969 & 2.2010 & 2.7181 & 3.1058 \\
\hline 12 & 0.6955 & 1.3562 & 1.7823 & 2.1788 & 2.6810 & 3.0545 \\
\hline 13 & 0.6938 & 1.3502 & 1.7700 & 2.1604 & 2.6503 & 3.0123 \\
\hline 14 & 0.6924 & 1.3450 & 1.7613 & 2.1448 & 2.6245 & 2.9768 \\
\hline 15 & 0.6912 & 1.3406 & 1.7531 & 2.1315 & 2.6025 & 2.0467 \\
\hline 16 & 0.6901 & 1.3368 & 1.7450 & 2.1189 & 2.5835 & 2.9208 \\
\hline 17 & 0.6892 & 1.3334 & 1.7396 & 2.1098 & 2.5669 & 2.8982 \\
\hline 18 & 0.6884 & 1.3304 & 1.7341 & 2.1009 & 2.5524 & 2.8784 \\
\hline 19 & 0.6876 & 1.3277 & 1.7291 & 2.0930 & 2.5395 & 2.8609 \\
\hline 20 & 0.6870 & 1.3253 & 1.7247 & 2.0860 & 2.5280 & 2.8453 \\
\hline 21 & 0.6864 & 1.3232 & 1.7207 & 2.0796 & 2.5176 & 2.8314 \\
\hline 22 & 0.6858 & 1.3212 & 1.7171 & 2.0739 & 2.5083 & 2.8188 \\
\hline 23 & 0.6853 & 1.3195 & 1.7139 & 2.0687 & 2.4899 & 2.8073 \\
\hline 24 & 0.6848 & 1.3178 & 1.7109 & 2.0639 & 2.4822 & 2.7970 \\
\hline 25 & 0.6844 & 1.3163 & 1.7081 & 2.0585 & 2.4851 & 2.7874 \\
\hline 28 & 0.6840 & 1.3150 & 1.7058 & 2.0555 & 2.4786 & 2.7787 \\
\hline 27 & 0.8837 & 1.3137 & 1.7033 & 2.0518 & 2.4727 & 2.7707 \\
\hline 28 & 0.6834 & 1.3125 & 1.7011 & 2.0484 & 2.4671 & 2.7633 \\
\hline 29 & 0.6830 & 1.3114 & 1. 6991 & 2.0452 & 2.4620 & 2.7564 \\
\hline 30 & 0.6828 & 1.3104 & 1.6973 & 2.0423 & 2.4573 & 2.7500 \\
\hline
\end{tabular}


Para estimar la media de muestras pequeñas $(\mathrm{n}<30)$, la fórmula a emplear es la siguiente:

$$
\text { IC } \mathrm{p}<0,05=\mathrm{X} \pm \mathrm{t} \cdot \mathrm{EEM}
$$

donde $\mathrm{t}$ es valor de la tabla $\mathrm{t}$ de Student para un nivel de significación estadística (error $\mathrm{p}>0,05$ o seguridad del 95\%), y con n-1 grados de libertad.

\subsection{Estimación de una proporción o porcentaje}

Se aplica cuando las variables del estudio son cualitativas o atributos, y en consecuencia, no podemos cuantificarlos para obtener su media aritmética.

Los pasos a seguir son idénticos que para la estimación de la media, con la única salvedad que al tratarse de un porcentaje (proporción), los resultados hallados tras una multirepetición del estudio, seguirían una distribución binomial.

Por tanto, lo primero será conocer el EE del porcentaje (EEP), que viene determinado por la siguiente fórmula:

$$
\operatorname{EEP}=\sqrt{P(1-P) /(n-1)}
$$

Una vez determinado el EEP, a continuación calculamos el IC con una seguridad del $95 \%$, o sea, un error menor del 5\% $(p<0,05)$. Para ello, basta con sumar y restar 1,96 veces el EEP al porcentaje obtenido del estudio.

IC $\mathrm{p}<0,05=\mathrm{P} \pm 1,96 \mathrm{EEP}=\mathrm{P} \pm 1,96 \cdot \sqrt{\mathrm{p}(1-\mathrm{p}) /(\mathrm{n}-1)}$

\section{Problemas resueltos}

Problema 1. La enfermera del trabajo de un servicio de prevención decide conocer cuál es la media del perímetro abdominal de su población trabajadora masculina mayor de 45 años. La población total de hombres con más de 45 años en su empresa es de 685 trabajadores. Para ello, decide analizar una muestra (aleatorizada y estratificada por edad) de 90 trabajadores con más de 45 años. El valor medio o media (x) de estas 90 mediciones fue de $89,6 \mathrm{~cm}$ y la desviación estándar (s) de 7,8 cm. ¿Cuál será el intervalo de confianza de la media $(\mu)$ de la población total masculina con una significación estadística menor del $5 \%$ ¿Cuáles serán los límites de confianza superior e inferior de dicho intervalo de confianza?

Paso $\mathrm{n}^{\mathrm{o}}$ 1. Determinar el EEM (Error Estándar de la Media). Para ello, aplicaremos la siguiente fórmula, donde $\mathrm{s}=7,8 \mathrm{~cm}$ y $\mathrm{n}=90$ trabajadores medidos.

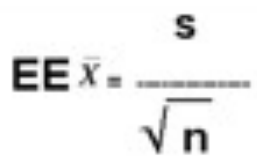

Entonces, $\mathrm{EEM}=0,82$

Paso $\mathrm{n}^{\circ}$ 2. Obtener el IC con un nivel de significación estadística $(\mathrm{p}<0,05)$, o sea, con un error menor del $5 \%$ o un nivel de confianza del $95 \%$. Aplicaremos la siguiente fórmula, donde $\mathrm{X}=89,6 \mathrm{~cm}$ y EEM=0,82

$$
\mathrm{X} \pm 1,96 \mathrm{EEM}
$$

Por tanto, $\mathrm{IC}(\mathrm{p}<0,05)=89,6 \pm 1,96(0,82)=89,6 \pm 1,6$

El Intervalo de confianza estaría formado por el siguiente rango:

IC $=88 \mathrm{~cm}-91,2 \mathrm{~cm}$, con un nivel de confianza del 95\%, que significa que, si esta enfermera repitiera el estudio 100 veces, en 95 ocasiones la media del perímetro abdominal estaría comprendido entre 88 y 91,2 cm. 
En cuanto a los límites de confianza, $88 \mathrm{~cm}$ sería el límite inferior y $91,2 \mathrm{~cm}$, el límite superior.

Podemos apoyarnos en herramientas estadísticas para estimar la media, como por ejemplo la hoja de cálculo de EXCEL:

Tendríamos que introducir los datos en una hoja de cálculo y a continuación, picar en la pestaña de fórmulas e insertar una función.

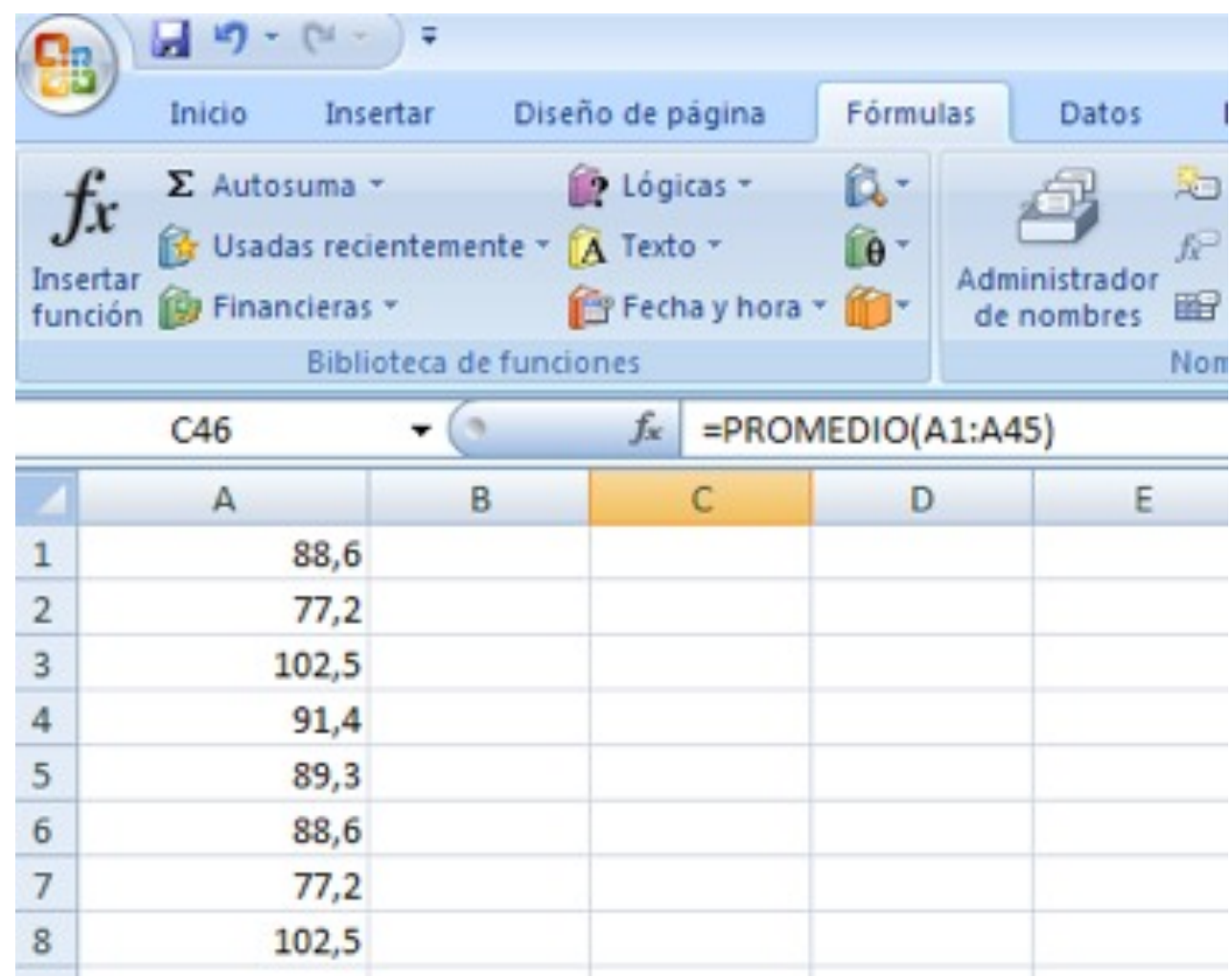

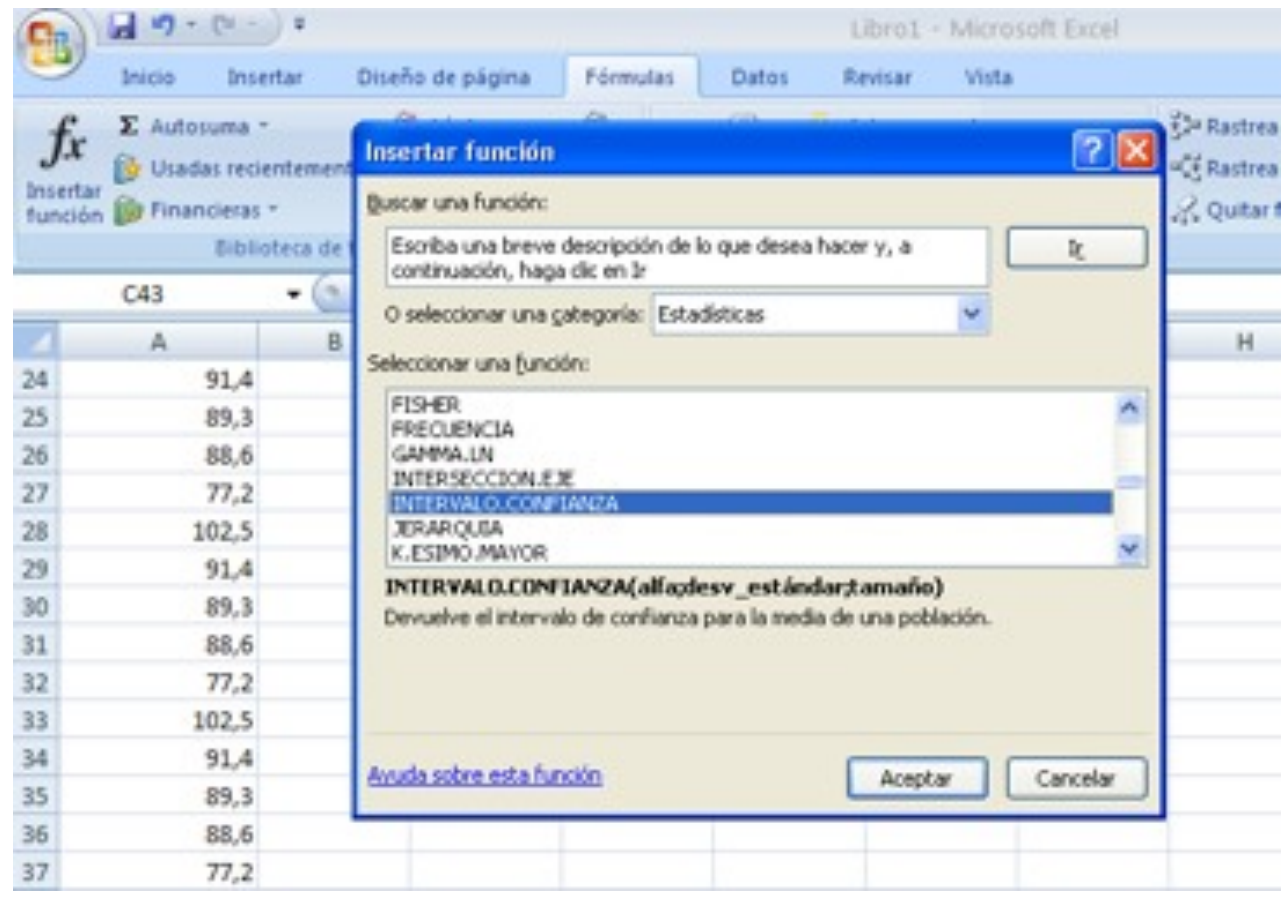




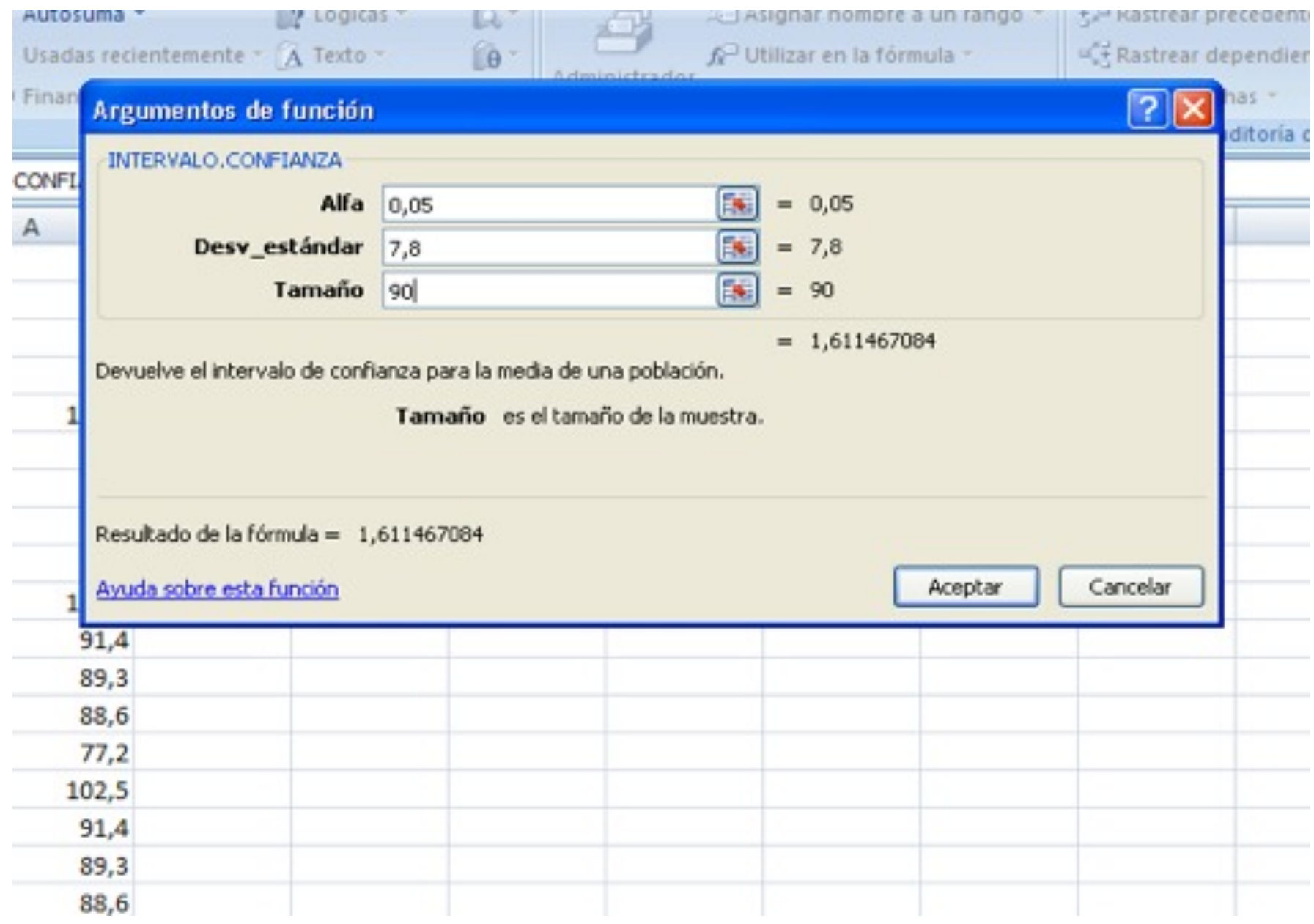

A continuación, introduciremos el nivel de significación estadística (alfa), el valor de la desviación estándar y el tamaño de la muestra analizada. El resultado aparece en la misma pantalla "Resultado de la fórmula=1,611..."

Problema 2. Un enfermero del trabajo de una unidad básica de salud desea conocer cuál es la media de antigüedad en el puesto de su población trabajadora femenina. En su empresa hay un total de 56 mujeres, y para ello decide tomar una muestra (aleatoria y estratifica) de 23 mujeres. La antigüedad media para esta muestra fue de 14,2 años y la desviación estándar de 6,9 años. ¿Cuál será el intervalo de confianza de la media $(\mu)$ de la población total de trabajadoras con una significación estadística menor del $5 \%$ ¿Cuáles serán los límites de confianza superior e inferior de dicho intervalo de confianza?

En este caso, el tamaño muestral es inferior a 30 observaciones, por tanto, se considera una muestra pequeña que sigue una distribución " $t$ " de Student. La fórmula que aplicaría nuestro compañero sería la siguiente:

$$
\text { IC } p<0,05=X \pm t \cdot E E M
$$

Paso ${ }^{\circ} 1$. El EEM se obtiene del mismo modo que en el caso anterior. Y así, el cálculo de EEM=1,44.

Paso $\mathrm{n}^{\mathrm{o}}$ 2. A continuación tendremos que conocer el valor del estadístico "t" que nos vendrá dado en la tabla de la distribución "t de Student". Los grados de libertad para esta muestra son (n-1), o sea, 22. Observando la tabla, buscaríamos en la columna de la izquierda los grados de libertad (22) y a continuación, en la fila superior, el nivel de significación estadística $(0,05)$. La intereseción de fila y columna nos daría la celda con el valor de $\mathrm{t}=1,7171$ 


\begin{tabular}{|c|c|c|c|c|c|c|}
\hline $\begin{array}{c}\text { Grados de } \\
\text { loertad }\end{array}$ & 0.25 & 0.1 & 0.05 & 0.025 & 0.01 & 0.005 \\
\hline 1 & 1.0000 & 3.0777 & 6.3137 & 12.7062 & 31.8210 & 63.6550 \\
\hline 2 & 0.8165 & 1.8856 & 2.8200 & 4.3027 & 6.8645 & 9.8250 \\
\hline 3 & 0.7649 & 1.6377 & 2.3534 & 3.1824 & 4.5407 & 5.8408 \\
\hline 4 & 0.7407 & 1.5332 & 2.1318 & 2.7705 & 3.7469 & 4.6041 \\
\hline 5 & 0.7267 & 1.4759 & 2.0150 & 2.5706 & 3.3049 & 4.0321 \\
\hline 6 & 0.7176 & 1.4398 & 1.8432 & 2.4460 & 3.1427 & 3.7074 \\
\hline 7 & 0.7111 & 1.4149 & 1.8946 & 2.3646 & 2.9979 & 3.4005 \\
\hline 8 & 0.7064 & 1.3908 & 1.8505 & 2.3060 & 2.8908 & 3.3584 \\
\hline$\theta$ & 0.7027 & 1.3830 & 1.8331 & 2.2622 & 2.8214 & 3.2498 \\
\hline 10 & 0.6008 & 1.3722 & 1.8125 & 2.2281 & 2.7638 & 3.1603 \\
\hline 11 & 0.0974 & 1.3834 & 1.7950 & 2.2010 & 2.7181 & 3.1058 \\
\hline 12 & 0.6055 & 1.3562 & 1.7823 & 2.1788 & 2.6810 & 3.0545 \\
\hline 13 & 0.6938 & 1.3502 & 1.7709 & 2.1004 & 2.6503 & 3.0123 \\
\hline 14 & 0.6924 & 1.3450 & 1.7613 & 2.1448 & 2.6245 & 2.9768 \\
\hline 15 & 0.6912 & 1.3406 & 1.7531 & 2.1315 & 2.6025 & 2.8467 \\
\hline 18 & 0.6901 & 1.3368 & 1.7450 & 2.1190 & 2.5835 & 2.8208 \\
\hline 17 & 0.6892 & 1.3334 & 1.7396 & 2.1098 & 2.5009 & 2.8982 \\
\hline 18 & 0.6884 & 1.3304 & 1.7341 & 2.1009 & 2.5524 & 2.8784 \\
\hline 10 & 0.6876 & 1.3277 & 1.7291 & 2.0930 & 2.5395 & 2.8000 \\
\hline 20 & 0.6870 & 1.3253 & 1.7247 & 2.0860 & 2.5280 & 2.8453 \\
\hline 21 & 0.6864 & 1.3232 & 1.7207 & 2.0796 & 2.5176 & 2.8314 \\
\hline 22 & 0.6858 & 1.3212 & 1.7171 & 2.0739 & 2.5083 & 2.8188 \\
\hline 23 & 0.6853 & 1.3185 & $7.7 / 139$ & 2.0087 & 2.4809 & 2.8073 \\
\hline 24 & 0.6848 & 1.3178 & 1.7100 & 2.0639 & 2.4922 & 2.7970 \\
\hline 25 & 0.6844 & 1.3163 & 1.7081 & 2.0505 & 2.4851 & 2.7874 \\
\hline 28 & 0.6840 & 1.3150 & 1.7058 & 2.0555 & 2.4786 & 2.7787 \\
\hline 27 & 0.6837 & 1.3137 & 1.7033 & 2.0518 & 2.4727 & 2.7707 \\
\hline 28 & 0.6834 & 1.3125 & 1.7011 & 2.0484 & 2.4671 & 2.7633 \\
\hline 29 & 0.6830 & 1.3114 & 1.0901 & 2.0452 & 2.4620 & 2.7564 \\
\hline 30 & 0.6828 & 1.3104 & 1.0973 & 2.0423 & 2.4573 & 2.7500 \\
\hline
\end{tabular}

Paso ${ }^{\circ} 3$. Determinar el IC.

$$
\text { IC } \mathrm{p}<0,05=\mathrm{X} \pm \mathrm{t} \cdot \mathrm{EEM}
$$

IC $\mathrm{p}<0,05=14,2 \pm 1,71(1,44)=14.2 \pm 2,46$

Límite inferior: 11,7

Límite superior: 16,7

Problema 3. Un enfermero del trabajo de una unidad básica de salud desea conocer cuál es la media de Hemoglobina Glicosilada (Hb A1c) de su población trabajadora. Para ello, decide tomar una muestra (aleatoria y estratificada) de 18 diabéticos. La media de Hb A1c obtenida fue de 8,1\% y la desviación estándar de 1,6. ¿Cuál será el intervalo de confianza de la media $(\mu)$ de la población total de trabajadoras con una significación estadística menor del 5\%? ¿Cuáles serán los límites de confianza superior e inferior del intervalo de confianza?

A pesar de que el valor está expresado en porcentaje, no debemos confundirlo con una variable cualitativa o atributo. Nuestra enfermera desea estimar la media de un parámentro (Hb Alc) que se expresa en valores porcentuales, pero que en realidad es una variable cuantitativa continua. 
$\mathrm{Al}$ igual que el caso anterior el tamaño muestral es inferior a 30 observaciones, por tanto, se considera una muestra pequeña que sigue una distribución " $t$ " de Student. La fórmula que aplicaría nuestro compañero sería la siguiente:

$$
\text { IC } p<0,05=X \pm t \cdot E E M
$$

Paso $\mathrm{n}^{\circ} 1$. El EEM se obtiene del mismo modo que en el caso anterior. Y así, el cálculo de EEM=0,39.

Paso $n^{\circ}$ 2. A continuación tendremos que conocer el valor del estadístico " $\mathrm{t}$ " que nos vendrá dado en la tabla de la distribución "t de Student". Los grados de libertad para esta muestra son (n-1), o sea, 17. Observando la tabla, buscaríamos en la columna de la izquierda los grados de libertad (17) y a continuación, en la fila superior, el nivel de significación estadística $(0,05)$. La intereseción de fila y columna nos daría la celda con el valor de $\mathrm{t}=1,7396$, o sea $\mathrm{t}=1,74$.

\begin{tabular}{|c|c|c|c|c|c|c|}
\hline $\begin{array}{c}\text { Grados de } \\
\text { loertad }\end{array}$ & 0.25 & 0.1 & 0.05 & 0.025 & 0.01 & 0.005 \\
\hline 1 & 1.0000 & 3.0777 & 6.3137 & 12.7002 & 31.8210 & 63.6550 \\
\hline 2 & 0.8165 & 1.8856 & 2.9200 & 4.3027 & 0.9645 & 9.9250 \\
\hline 3 & $0.704 \theta$ & 1.0377 & 2.3534 & 3.1824 & 4.5407 & 5.8408 \\
\hline 4 & 0.7407 & 1.5332 & 2.1318 & 2.7705 & 3.7460 & 4.0041 \\
\hline 5 & 0.7267 & 1.4750 & 2.0150 & 2.5706 & 3.3640 & 4.0321 \\
\hline 6 & 0.7176 & 1.4398 & 1.8432 & 2.4400 & 3.1427 & 3.7074 \\
\hline 7 & 0.7111 & 1.4148 & 1.8948 & 2.3646 & 2.8979 & 3.4895 \\
\hline 8 & 0.7084 & 1.3968 & 1.8505 & 2.3000 & 2.8965 & 3.3554 \\
\hline 9 & 0.7027 & 1.3830 & 1.8331 & 2.2022 & 2.8214 & 3.2498 \\
\hline 10 & 0.0908 & 1.3722 & 1.8125 & 2.2281 & 2.7638 & 3.1093 \\
\hline 11 & 0.6974 & 1.3634 & 1.7950 & 2.2010 & 2.7181 & 3.1088 \\
\hline 12 & 0.6055 & 1.3502 & 1.7823 & 2.1788 & 2.6810 & 3.0545 \\
\hline 13 & 0.6038 & 1.3502 & 1.7709 & 2.1004 & 2.0503 & 3.0123 \\
\hline 14 & 0.0024 & 1.3450 & 1.7613 & 2.1448 & 2.6245 & 2.8708 \\
\hline 15 & 0.0912 & 1.3406 & 1.7531 & 2.1315 & 2.8025 & 2.8487 \\
\hline 16 & 0.6901 & 1.3308 & 1.7459 & 2.1180 & 2.5835 & 2.9208 \\
\hline 17 & 0.6892 & 1.3334 & 1.7390 & 2.1088 & 2.5000 & 2.8982 \\
\hline 18 & 0.6884 & 1.3304 & 1.7341 & 2.1000 & 2.5524 & 2.8784 \\
\hline 19 & 0.8876 & 1.3277 & 1.7291 & 2.0930 & 2.5395 & 2.8009 \\
\hline 20 & 0.6870 & 1.3253 & 1.7247 & 2.0800 & 2.5280 & 2.8453 \\
\hline 21 & 0.6804 & 1.3232 & 1.7207 & 2.0796 & 2.5176 & 2.8314 \\
\hline 22 & 0.6858 & 1.3212 & 1.7171 & 2.0730 & 2.5083 & 2.8188 \\
\hline 23 & 0.6853 & 1.3195 & 1.7130 & 2.0087 & 2.4900 & 2.8073 \\
\hline 24 & 0.6848 & 1.3178 & 1.7100 & 2.0039 & 2.49222 & 2.7970 \\
\hline 25 & 0.6844 & 1.3163 & 1.7081 & 2.0595 & 2.4851 & 2.7874 \\
\hline 26 & 0.6840 & 1.3150 & 1.7056 & 2.0555 & 2.4786 & 2.7787 \\
\hline 27 & 0.6837 & 1.3137 & 1.7033 & 2.0518 & 2.4727 & 2.7707 \\
\hline 28 & 0.6834 & 1.3125 & 1.7011 & 2.0484 & 2.4671 & 2.7633 \\
\hline 20 & 0.6830 & 1.3114 & 1.8001 & 2.0452 & 2.4620 & 2.7564 \\
\hline 30 & 0.6828 & 1.3104 & 1.6973 & 2.0423 & 2.4573 & 2.7500 \\
\hline
\end{tabular}

Problema $n^{0}$ 4. Una enfermera del trabajo desea conocer cuál es la prevalencia de diabetes en su empresa. Para ello, decide tomar una muestra (aleatoria y estratificada) de 250 trabajadores y determinar su glucemia basal. La prevalencia obtenida fue del $6,2 \%$. ¿Cuál será la prevalencia de la población general de trabajadoras con una significación estadística menor del 5\%? ¿Cuáles serán los límites de confianza superior e inferior del intervalo de confianza? 
La fórmula que aplicará nuestra compañera en este estudio es la siguiente:

$$
\operatorname{EEP}=\sqrt{P(1-P) /(n-1)}
$$

Donde $\mathrm{P}=6,2 \%, \mathrm{y} n=250$, entonces:

$\mathrm{EEP}=\sqrt{ } 0,062(1-0,062) / 249=0,015$

IC $\mathrm{p}<0,05=\mathrm{P} \pm 1,96$ EEP

IC $p<0,05=6,2 \% \pm 1,96(0,015)=6,2 \% \pm 0.03 \%$

Límite Inferior: 5,9\%

Límite Superior: 6,5\% 